\title{
An Enhanced Co-Scheduling Method using Reduced MS-State Diagrams
}

\author{
R. Govindarajan \\ Supercomputer Edn. \& Res. Centre \\ Computer Science \& Automation \\ Indian Institute of Science \\ Bangalore 560 012, India \\ govind@ $\{$ serc,csa $\}$.iisc.ernet.in
}

\author{
N.S.S. Narasimha Rao \\ Novell Software \\ Development India Ltd. \\ Garvephavipalya \\ Bangalore 560 068, India \\ nnarasimharao@novell.com
}

\author{
E.R. Altman \\ IBM T.J. Watson \\ Research Center \\ Yorktown Heights \\ NY 10598, U.S.A. \\ erik@watson.ibm.com
}

\author{
Guang R. Gao \\ Electrical \& Computer Engg. \\ University of Delaware \\ Newark \\ DE 19716, U.S.A \\ ggao@eecis.udel.edu
}

\begin{abstract}
Instruction scheduling methods based on the construction of state diagrams (or automata) have been used for architectures involving deeply pipelined function units. However, the size of the state diagram is prohibitively large, resulting in high execution time and space requirement.

In this paper, we present a simple method for reducing the size of the state diagram by recognizing unique paths of a state diagram. Our experiments show that the number of paths in the reduced state diagram is significantly lower by 1 to 3 orders of magnitude - compared to the number of paths in the original state diagram.

Using the reduced MS-state diagrams, we develop an efficient software pipelining method. The proposed software pipelining algorithm produced efficient schedules and performed better than Huff's Slack Scheduling method, and the original Co-scheduling method, in terms of both the initiation interval (II) and the time taken to construct the schedule.
\end{abstract}

\section{Introduction}

Function units in modern architectures involve increasingly more complex resource usage. Deeper pipelines and wide-word instructions are staging a resurgence. Processors capable of issuing 8 instructions per cycle are on the horizon, and structural hazard resolution is expected to be more complex. With such complex resource usage, the instruction scheduler must check and avoid any structural hazard, e.g., contention for hardware resources by instructions. Conventional instruction scheduling and software pipelining methods $[4,6,7,10,12,13,17,18]$ accomplish this by maintaining a global resource table (or Modulo Reservation Table (MRT) in the case of software pipelining) to model the resource usage. In these methods instructions are scheduled using a naive method, attempting to schedule operations at each time slot until a conflict-free slot is found. Further, the approach followed here is greedy and involves several tries and retries.

Recently, a finite state automaton (FSA)-based instruction scheduling technique $[2,14,16]$, which uses the notion of forbidden/permissible latencies, and legal initiation sequences $[3,11,15]$ to efficiently detect resource conflict has been proposed. The FSA-based methods have effectively reduced the problem of checking structural hazards to a fast table lookup. The Co-scheduling framework proposed by us in [8] is a software pipelining method that makes use of classical pipeline theory and state diagram construction. The constructed state diagram, called a Modulo-Scheduled (MS)-state diagram, represents valid latency sequences. Each path in the MS-state diagram corresponds to a set of time steps at which different instructions can be initiated in the given pipeline under modulo scheduling without incurring any structural hazard. In the Co-scheduling method, a single path in the MS-state diagram, and the time steps corresponding to it are used to guide the software pipelining method.

Unfortunately, the practical use of state diagram based instruction scheduling methods $[2,8,14,16]$ is restricted due to the large size of the state diagram. In the context of Co-scheduling, the MS-state diagram may consist of a large number of paths especially for large values of the loop initiation interval (II). One basic observation that we make in this paper is that a significant number of paths in a MSstate diagram are redundant. A simple method that detects unique paths, known as primary paths, has been presented in this paper. A state diagram consisting only primary paths is termed as a reduced state diagram. Our experiments reveal a significant reduction - by 1 to 3 orders of magnitude - in the number of paths in the reduced MS-state diagram, Further, we have proposed an alternative direct method to derive the scheduling information represented by the reduced state diagram. The direct method proposed in this paper is faster, by a factor of 2 to 3 , in terms of ex- 
ecution time compared to the construction of the reduced MS-state diagram.

In this paper, the original Co-scheduling method is also enhanced by considering multiple paths of the MS-state diagram and choosing the most appropriate one based on the data dependences in the loop. The enhanced Co-scheduling method has been tested on a large number of loop kernels taken from several benchmark programs. The use of multiple offset sets and the reduced state diagrams facilitate achieving better software pipelined schedules with smaller II and shortens the scheduling time. Further, comparison with Huff's Slack Scheduling [10] reveals a 4-fold (or 400\%) improvement in the time taken to construct the schedule. Further, the enhanced Co-scheduling method results in a moderate improvement in terms of II, particularly in loops which are resource-critical. Lastly, the enhanced Co-scheduling method also produces better schedules compared to original Co-scheduling [8] in terms of both II and the time to construct the schedule.

In the following section we motivate the need for reduced state diagram and enhanced Co-scheduling. In Section 3, we present the underlying theory of reduced MSstate diagrams. Section 4 deals with the construction of reduced MS-state diagrams and the enhanced Co-scheduling algorithm. In Section 5, we report the results of our experiments. Related works are compared in Section 6 and concluding remarks are presented in Section 7.

\section{Background and Motivation}

In this section we present the necessary background on Co-scheduling. Throughout this paper, we use the terms, "operations", "instructions", and "initiations" synonymously. We assume that our architecture consists of multiple functional units (FUs), where each FU is capable of executing instructions of a specific instruction class. Further, each FU is a static pipeline whose resource usage pattern is described by a single reservation table [11]. ${ }^{1}$

\subsection{Modulo-Scheduled State Diagram}

The latency sequences that do not cause structural hazards in a pipeline operating under modulo scheduling are represented in a Modulo-Scheduled (MS) state diagram $[8,9]$. The reservation table of an FU and its MSstate diagram for an $\mathbf{I I}=6$ are shown in Figure 1. A path $S_{0} \stackrel{p_{1}}{\longrightarrow} S_{1} \stackrel{p_{2}}{\longrightarrow} S_{2} \cdots \stackrel{p_{k}}{\longrightarrow} S_{k}$ in the MS-state diagram corresponds to a latency sequence $\left\{p_{1}, p_{2}, \cdots, p_{k}\right\}$. The latency sequence represents $k+1$ initiations that are made at time steps $0, p_{1},\left(p_{1}+p_{2}\right), \cdots,\left(p_{1}+p_{2}+\cdots+p_{k}\right)$.

\footnotetext{
${ }^{1}$ It is possible to extend the work presented in this paper to architectures in which the pipelines share certain resources, such as the result write bus. A discussion on this is beyond the scope of this paper.
}

In modulo scheduling, these time steps correspond to the offset values

$$
0, p_{1},\left(p_{1} \oplus p_{2}\right), \cdots,\left(p_{1} \oplus p_{2} \oplus \cdots \oplus p_{k}\right),
$$

where $\oplus$ corresponds to addition modulo II,

As an example, the latency sequence $\{3\}$ corresponding to the path $S_{0} \stackrel{3}{\longrightarrow} S_{3}$ represents only 2 initiations made at time steps 0 and 3 . Thus analyzing the MS-state diagram reveals valid latency sequences and the corresponding offset sets that do not cause collision. Further, in this state diagram paths $S_{0} \stackrel{2}{\longrightarrow} S_{1} \stackrel{2}{\longrightarrow} S_{3}$ and $S_{0} \stackrel{4}{\longrightarrow} S_{2} \stackrel{4}{\longrightarrow} S_{3}$ result in maximum number of initiations in the pipeline. We refer to $\{2,2\}$ and $\{4,4\}$ as maximum initiation latency sequences.

\subsection{Co-scheduling Method}

Co-scheduling was based on Huff's Slack Scheduling algorithm [10]. Co-scheduling starts with a Minimum Initiation Interval (MII) and attempts to schedule the loop for values of II $\geq$ MII until a schedule is found. For a given II it computes the MS-state diagram for all FUs that have structural hazards. To make the discussion concise and address only the relevant points, we will assume that there is only one type of pipeline ${ }^{2}$. From the MS-state diagram for the pipeline, a maximum initiation latency sequence is derived.

The basic notion of Huff's original Slack Scheduling [10] was to schedule instructions in increasing order of their slackness: the difference between the earliest time and the latest time at which an instruction may be scheduled. Slack is a dynamic measure and is updated after each instruction is scheduled. These points remain in Coscheduling. The difference lies in how a time slot is chosen within the slack range. The original Slack Scheduling method attempts to schedule an instruction in every time step it its slack range until it finds a conflict-free slot. The Co-scheduling method, however, attempts to schedule an instruction only at pre-determined offset values (in the slack range) given by the maximum initiation latency sequence.

To clarify matters, consider the motivating example (Figure 1). Suppose we have chosen the maximum initiation latency sequence $\{2,2\}$ and the corresponding offset values are 0,2 , and 4 . Further let us assume an instruction $i_{1}$ is scheduled at time step, say, 1 and two more instructions $i_{2}$, and $i_{3}$ are to be scheduled in the same pipeline. If the slack of $i_{2}$ is $(4,6)$, i.e., instruction $i_{2}$ can be placed at any time step from 4 to 6 , the Co-scheduling method will

\footnotetext{
${ }^{2}$ This assumption (only one type of pipeline) is made only to simplify the discussion. Neither the original Co-scheduling method nor the enhanced Co-scheduling method presented in this paper is restricted by this.
} 


\begin{tabular}{|c|c|c|c|c|c|c|}
\hline \multirow{2}{*}{ Stage } & \multicolumn{7}{|c|}{ Time Steps } \\
\hline & 0 & 1 & 2 & 3 & 4 & 5 \\
\hline 1 & $\mathrm{x}$ & & & & & \\
\hline 2 & & $\mathrm{x}$ & $\mathrm{x}$ & & & \\
\hline 3 & & & & $\mathrm{x}$ & & \\
\hline
\end{tabular}

(a) Cyclic Reservation Table

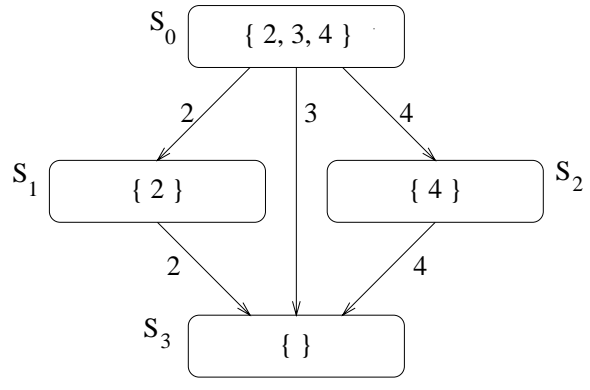

(b)

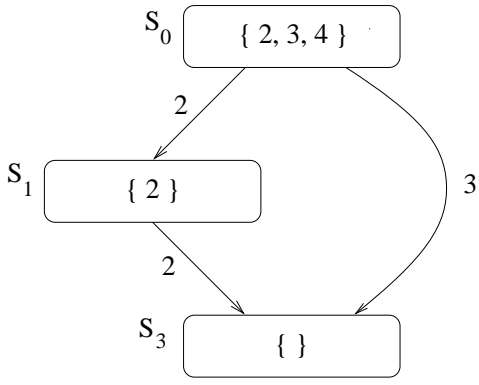

(c)

Figure 1: Full and Reduced MS-State Diagrams for Example Reservation Table

choose time step 5 , so that the offset between $i_{1}$ and $i_{2}$ is 4 , as governed by the chosen latency sequence.

The Co-scheduling method goes by the chosen maximum initiation latency sequence, even if this means avoiding some other latency values that would yield a legal partial schedule. Note that, in the considered example, even though $i_{2}$ can be scheduled at time step 4, with a (permissible) latency 3 with $i_{1}$, the Co-scheduling method does not attempt this. This is because if $i_{1}$ and $i_{2}$ are scheduled at times 1 and 4 (offsets 0 and 3) respectively, then instruction $i_{3}$ cannot be scheduled in the same pipe. Thus the Co-scheduling method takes a global view in scheduling instructions and deals with structural hazards much more efficiently, by using a maximum permissible latency sequence.

\subsection{Reduced State Diagram: Motivation}

The use of a single offset set, as done in Co-scheduling, may not be sufficient to obtain a schedule with the lowest value of II. We illustrate this with our example discussed in Section 2.2. Suppose there are only two instructions $i_{1}$ and $i_{2}$. Further assume that instructions $i_{1}$ and $i_{2}$ have a tight slack, requiring them to be scheduled at time steps 1 and 4 respectively. If we use a single set of offset values, e.g., 0, 2, and 4 , as suggested by the original Co-scheduling, then one of the two instructions cannot be scheduled in the pipeline, even though the chosen latency sequence can accommodate 3 operations in the pipeline. In this case, eventually the Coscheduling algorithm will result in an II of 7. However, if we had used the latency sequence $\{3\}$, or the corresponding offset values 0 and 3 , then we could have scheduled the loop with II $=6$. Thus, it is beneficial, if Co-scheduling can consider all offset sets.

The number of offset sets for an MS-state diagram can be quite large (in the order of several 100,000s). Further, the number of paths in an MS-state diagram increases drastically for large values of II. As a consequence, the con- struction of the state diagram is expensive in terms of both space and time complexity, especially for large II values.

Consider the state diagram shown in Figure 1(b). Clearly, the states $S_{0}, S_{1}, S_{2}$, and $S_{3}$ are all distinct. However, the information represented by the paths $S_{0} \stackrel{2}{\longrightarrow}$ $S_{1} \stackrel{2}{\longrightarrow} S_{3}$ and $S_{0} \stackrel{4}{\longrightarrow} S_{2} \stackrel{4}{\longrightarrow} S_{3}$ are not. Though the latency sequences corresponding to the above paths are different, it can be seen that the offset values for both of them are 0,2 , and 4 . Thus the latter path (actually any one of the two paths) is redundant. This raises the question, that even though state $S_{2}$ is distinct, can we avoid generating the state if all the paths that go through $S_{2}$ are redundant. Equivalently, can we list only the paths that lead to distinct offset sets? In our example, removing state $S_{2}$ and the arcs that are connected to it, does not result in any loss of information. The reduced state diagram is shown in Figure 1(c).

In this paper, we develop a method to construct the reduced MS-state diagram which consists of only unique paths. In Section 4, an enhanced Co-scheduling algorithm that uses many latency sequences, not just a maximum initiation latency sequence.

\section{Reduced MS-State Diagrams}

We begin with a few definitions. States $S_{0}$ and $S_{f}$ correspond to start and final states in the MS-state diagram.

Definition 3.1 A path $S_{0} \stackrel{p_{1}}{\longrightarrow} S_{1} \stackrel{p_{2}}{\longrightarrow} S_{2} \ldots \stackrel{p_{k}}{\longrightarrow} S_{f}$ in the MS-state diagram is called primary if the sum of the latency values does not exceed II. That is, $p_{1}+p_{2}+\cdots+$ $p_{k}<$ II. A path is called secondary if $p_{1}+p_{2}+\cdots+p_{k}>$ II.

Note that the sum of the latencies along any path in the MSstate diagram will not be equal to II. Otherwise, the initiation representing state $S_{f}$ corresponds to the offset value 0 which causes a collision with the initiation at $S_{0}$ (the initial state). 
Definition 3.2 The set of permissible offsets $\mathcal{O}$ equals the set of all initial offset values at which an initiation is permitted.

For the FU discussed in Section 2 (refer to Figure 1), the set of permissible offsets is $\{0,2,3,4\}$. Next, we adapt the following definitions from $[11,15]$.

Definition 3.3 Two offsets $o_{1}$ and $o_{2}$ belonging to $\mathcal{O}$ are compatible if $\left(o_{1}-o_{2}\right) \bmod \mathbf{I I}$ is in $\mathcal{O}$.

Definition 3.4 A compatibility class with respect to $\mathcal{O}$ is a set in which all pairs of elements are compatible.

Definition 3.5 A maximal compatibility class is a compatibility class that is not a proper subset of any other compatible class.

Two compatible classes for $\{0,2,3,4\}$ are $\{0,2,4\}$ and $\{0,3\}$. These compatibility classes are maximal, while $\{0,2\}$ is not.

The compatibility classes of $\mathcal{O}$ are related to the offset sets of different paths in the MS-state diagram. The following lemmas establish that. The reader is referred to [9] for proofs of lemmas and theorems.

Lemma 3.1 The offset set of any path from the start state $S_{0}$ to the final state $S_{f}$ in the MS-state diagram forms a maximal compatibility class of $\mathcal{O}$.

Lemma 3.2 For each maximal compatibility class $C$ of permissible offsets, there exists a primary path in the MSstate diagrams whose offset set $O$ is equal to $C$.

Using the above two lemmas, we can establish that:

Theorem 3.1 For each secondary path from $S_{0}$ to $S_{f}$ in the MS-state diagram there exists a primary path such that their offset sets are equal.

Theorem 3.2 A reduced MS-state diagram consisting only of primary paths contains the set of all valid offset sets that are permissible in the original state diagram.

From the above theorems, it can be seen that though the number of paths in the original state diagram for either a fully pipelined or non-pipelined function unit is large, there is only one primary path (or one distinct offset set) in the reduced state diagram.

\section{Enhanced Co-Scheduling using Reduced MS-State Diagram}

In this section, we present two methods to obtain the unique offset sets. Section 4.3 deals with the enhanced Coscheduling method.

\subsection{Generation of Offset Sets using Reduced MS- State diagram}

As demonstrated in Section 3 (Theorem 3.2), it is sufficient to obtain a reduced MS-state diagram consisting only of primary paths. Thus, one method to obtain a reduced state diagram is by identifying secondary paths and eliminating them. This is done two phases. In the first phase, at the time of creation of a new state, the state diagram construction method checks whether the path leading to the newly created state is secondary. If so, it marks such a state as Redundant and the construction of the subtrees of the state is stopped. In the second phase, the algorithm checks each state for redundant children and removes them. If all the children of a state are redundant, then the state itself is marked redundant. Subsequently, this state gets eliminated in the recursive ascend, that is, when its parent is checked for redundant children.

\subsection{Generation of Offset Sets using Maximal Compatible Classes}

Lemmas 3.1 and 3.2 establish the correspondence between the offset sets of primary paths and maximal compatible classes of the permissible offset set. Hence obtaining all maximal compatible classes is an alternative way of obtaining the offset sets. An approach to obtain the compatible classes is presented in [11] (page 99) ${ }^{3}$. This approach is a direct way of obtaining the set of all offset sets of the reduced MS-state diagram. We compare the performance of the offset set generation using the two methods, viz., reduced MS-state diagrams and maximal compatible classes, in Section 5.2. It should, however, be noted that the software pipelining algorithm presented in the following section is independent of the method used to obtain the set of offsets.

\subsection{Enhanced Co-Scheduling Algorithm}

Like Slack Scheduling algorithm, Co-scheduling uses (dynamic) slack-based priority, and stretchability measure to determine whether to the schedule an operation early or late in order to reduce the register pressure [10]. The main difference between the two methods is the way a conflictfree slot is chosen for scheduling an operation. For this, we consider all offset sets that have a cardinality greater than or equal to the number of operations in the loop that are mapped on to that function unit ${ }^{4}$. Sets with smaller

\footnotetext{
${ }^{3}$ It is important to note here that the generation of compatible classes was discussed in classical pipeline theory in the context of reconfiguring pipelines rather than for analyzing pipelines.

${ }^{4}$ For simplicity, we consider only a single instance of FU in each FU type.
} 
cardinalities, need not be considered as they do not support the required number of instructions. The way scheduling proceeds is better explained with the help of an example. Though the example concentrates on how resource constraints are met in a single FU, the method is general enough to handle multiple FU types. The reader is referred to [9] for the detailed algorithm.

Consider, a function unit with four instructions $i_{1}, i_{2}, i_{3}$, and $i_{4}$ mapped on to it. Consider the following offset sets which support four or more initiations:

$$
O_{1}=\{0,1,7,10\} ; O_{2}=\{0,1,6,7\} ; O_{3}=\{0,3,6,9,12\} ;
$$

Initially, the sets $O_{1}, O_{2}$, and $O_{3}$ are the active offset sets. Let us start with the scheduling of instruction $i_{1}$ with a slack $(3,5)$. Since this is the first instruction to be scheduled in the pipe, it has no structural hazards and can be placed in any cycle in its slack. To simplify the discussion, it is assumed that all instructions are placed as early as possible. Hence $i_{1}$ is placed at time 3 .

Since offset values are relative, the first instruction is always assumed to be scheduled at an offset 0 . In our example, instruction $i_{1}$ which is scheduled at time step 3 corresponds to an offset 0 . Now, suppose $i_{2}$ has a slack $(10,15)$. The Estart time of $i_{2}$ corresponds to an offset $10-3=7$ with respect to $i_{1}$. A look at the offset sets reveals that $7,9,10$, and 12 are permissible offsets. Hence $i_{2}$ is scheduled at time step 10 with an offset 7 with respect to $i_{1}$. Since $O_{3}$ does not support an offset 7 , it is marked inactive; the offset sets $O_{1}$ and $O_{2}$ are currently active.

Now, if instruction $i_{3}$ has a tight slack $(12,12)$ with an offset 9, neither of the offset sets $O_{1}$ and $O_{2}$ can support the scheduling of $i_{3}$ at time 12. In such a case, the most recently placed operation is ejected. This may increase the number of active offset sets and hence the possibility of a placement. In our example, when $i_{2}$ is unscheduled, the offset set $O_{3}$ becomes active, and $i_{3}$ can be scheduled at time 12 (with offset 9). However, this makes offset sets $O_{1}$ and $\mathrm{O}_{2}$ inactive, leaving $\mathrm{O}_{3}$ as the only active set. Subsequently, when $i_{2}$ is chosen for scheduling ${ }^{5}$, it is scheduled at time step 15 , with offset 12 . In a similar manner, if instruction $i_{4}$ has a slack $(19,26)$, it can be placed at one of the remaining offsets, 3 or 6 . If no valid schedule is found even after ejecting a number of operations (greater than a threshold value), the current (partial) schedule is aborted and successive values of II are tried until a valid schedule is obtained.

In addition to the difference in selecting the schedule slot for an operation, enhanced Co-scheduling differs from Huff's Slack Scheduling in one other way. In forcing the placement of an operation, Huff's method ejects only conflicting operations; whereas in our approach, the operations scheduled in an FU are ejected in the reverse order in which

\footnotetext{
${ }^{5}$ For simplicity, assume that $i_{2}$ 's slack does not change.
}

they are scheduled. This is in order to ensure that our method does not get stuck with an active offset set. Enhanced Co-scheduling extends the original Co-scheduling method to consider multiple offset sets, and thus providing more opportunities for scheduling the loop.

\section{Experimental Results}

In this section, we present quantitative results for reduced MS-state diagrams and the enhanced Co-scheduling method.

\subsection{Reduced MS-state Diagram}

First we compare the number of paths in the reduced MS-state diagram with that in the original state diagram. In order to exercise our state diagram-based method fully, for the 6 functional units considered in our experiments, we chose long reservation tables (representing deeper pipelines) with arbitrary structural hazards. ${ }^{6}$

\begin{tabular}{|l|c|c|c|c|c|c|}
\hline $\begin{array}{l}\text { Average } \\
\text { Reduction } \\
\text { in \# Paths }\end{array}$ & FU-1 & FU-2 & FU-3 & FU-4 & FU-5 & FU-6 \\
\hline \multirow{8}{*}{ Geo. Mean } & 7.5 & 20.8 & 2.5 & 2.6 & 26.1 & 2.2 \\
\cline { 2 - 7 } & & 60.7 & 3.0 & 3.4 & 64.7 & 2.5 \\
\hline Arith. Mean & 13.5 & $16 \leq \mathbf{I I} \leq 24$ \\
\hline \multirow{2}{*}{ Geo. Mean } & 1037.3 & 9084.3 & 54.3 & 52.3 & 2273.9 & 32.7 \\
\hline Arith. Mean & 3882.5 & 27543.7 & 99.5 & 95.3 & 22490.7 & 55.5 \\
\hline
\end{tabular}

Table 1: Average Reduction in the Number of Paths.

Table 1 shows the average reduction in the number of paths achieved by considering only the primary paths, the average being taken over different values of II from 8 to $24 .{ }^{7}$ It can be seen that especially for large values of II, between 16 to 24 , the reduction in number of paths is very significant (the geometric mean varies from 32 to 9,084). This can be further verified from the rate of increase in the number of paths for the original and reduced state diagrams plotted for FU-1 and FU-3 in Figure 2. Note that the y-axis (number of paths) in Figure 2 is on a log-scale. Lastly, for functional units FU-3, FU-4, and FU-6, the reduction in the number of paths is not so significant for the values of II considered in our experiments. This is because, at small II values, many of the latencies were forbidden for these FUs.

\footnotetext{
${ }^{6}$ The reservation tables of the functional units used in this experiment and in the enhanced Co-scheduling method are listed in [9] which is available over the internet.

${ }^{7}$ For values of II greater than 24 , the number of paths in the original state diagram exceeds 10 Millions and all paths could not be enumerated within 30 minutes of CPU time. However, the reduced MS-state diagram has been constructed even for large values of II (upto 85).
} 


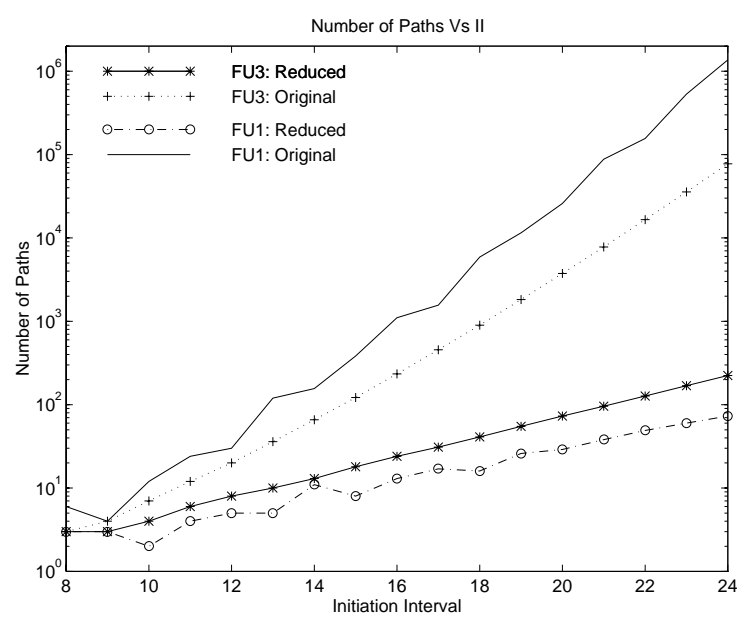

Figure 2: Number of Paths vs. II

\subsection{Maximal Compatible Set Generation}

Next we compare the reduced state-diagram approach for generating the offset sets with the direct approach of enumerating maximal compatibility classes. For the same set of reservation tables used in the previous section, and for the same II values, we compared the execution time, on an UltraSparc 170E, of the two approaches. The average execution time, averaged over runs for different values of II, for each FU are shown in Table 2. Obtaining the offset sets from the maximal compatible classes results in a 3 -fold improvement in execution time for the values of II considered in our experiments.

\begin{tabular}{|l|c|c|c|c|c|c|}
\hline \multirow{2}{*}{ Approach } & \multicolumn{6}{|c|}{ Avg. Exec. Time } \\
\cline { 2 - 7 } & FU-1 & FU-2 & FU-3 & FU-4 & FU-5 & FU-6 \\
\hline $\begin{array}{l}\text { Red. State Diag. } \\
\text { (Arith. Mean) }\end{array}$ & 277.7 & 1025.2 & 80.8 & 2933.8 & 138.2 & 17.6 \\
\hline $\begin{array}{l}\text { Comp. Class } \\
\text { (Arith. Mean) }\end{array}$ & 70.7 & 146.1 & 93.1 & 519.4 & 74.5 & 7.0 \\
\hline \hline $\begin{array}{l}\text { Improvement } \\
\text { (Geo. Mean) }\end{array}$ & 6.9 & 5.3 & 1.9 & 3.8 & 3.1 & 3.7 \\
\hline
\end{tabular}

Table 2: Execution Time of Two Methods to Generate Offset Sets

\subsection{Performance of Enhanced Co-Scheduling Method}

We experimented the enhanced Co-scheduling method on 1153 loops taken from a variety of scientific benchmarks. We assumed a target architecture with 7 function units: 2 Integer, 1 Load, 1 Store, 1 FP Add, 1 FP Multiply, and 1 FP Divide Units, each having complex resource usage. The reservation tables for the FP Multiply and FP Add Units correspond to those of FU-5 and FU-6, respectively, used in Sections 5.1 and 5.2.

\begin{tabular}{|l|c|c|c|c|c|c|c|}
\hline \multirow{2}{*}{} & \multicolumn{7}{|c|}{ II - MII } \\
\cline { 2 - 8 } & 0 & 1 & 2 & 3 & 4 & 5 & $\geq 6$ \\
\hline No. of Cases & 880 & 144 & 20 & 17 & 15 & 61 & 16 \\
\hline \% Cases & 76.3 & 12.5 & 1.7 & 1.5 & 1.3 & 5.3 & 1.4 \\
\hline
\end{tabular}

Table 3: Performance of Enhanced Co-Scheduling

Table 3 gives a break up of the total benchmark programs in terms of how far the II of the constructed schedule is from the minimum initiation interval (MII). Our enhanced Co-scheduling found schedules at the minimum initiation interval in 880 cases. In the remaining cases, II of the resulting schedules was 2.58 time steps away, on an average, from the minimum initiation interval. The (arithmetic) mean time to compute a schedule is 2.9 milliseconds, while the median for this is 1.1 milliseconds. The time to construct a schedule does not include the time to construct reduced MS-state diagrams for different function units. This is because, the generation of the offset sets can be done off-line, and stored in a database. Lastly, even though enhanced Co-scheduling uses the set of all offset sets, typically several hundreds in number, it still was successful in finding a schedule within a few milliseconds.

\subsection{Comparison with Slack Scheduling}

The enhanced Co-scheduling method is compared with our implementation of Huff's Slack Scheduling method ${ }^{8}$. The results of our comparison are presented in Table 4. The term percentage improvement for various quantities, such as II, is calculated for each loop as follows:

$$
\begin{aligned}
& \% \text { Impr in II } \\
& \text { for Method A }
\end{aligned}=\frac{I I_{\text {Method B }}-I I_{\text {Method A }}}{I I_{\text {Method A }}} * 100
$$

The numbers reported in columns 4 and 7 in Tables 4 and 5 are averaged over all loops reported in columns 2 and 5 respectively.

As seen from Table 4 our enhanced Co-scheduling results in better II in 114 benchmarks; the average improvement in II is $13.5 \%$. In a large number of cases ( 993 benchmarks) both methods achieved the same II. This is because, for the target architecture considered for the scheduling, only one-fourth (24\%) of the loops are resource-critical i.e., resource MII (ResMII) dominates recurrence MII

\footnotetext{
${ }^{8}$ To the best of our knowledge, our implementation of Huff's Slack Scheduling method faithfully follows the implementation details presented in [10].
} 


\begin{tabular}{|c|c|c|c|c|c|c|c|c|}
\hline \multirow[t]{2}{*}{ Measure } & \multicolumn{3}{|c|}{ Enhanced Co-Scheduling Better } & \multicolumn{3}{|c|}{ Huff Better } & \multicolumn{2}{|c|}{ Both Same } \\
\hline & $\begin{array}{c}\text { No. of } \\
\text { Benchmarks }\end{array}$ & $\%$ Cases & $\begin{array}{c}\% \text { Improve- } \\
\text { ment }\end{array}$ & $\begin{array}{c}\text { No. of } \\
\text { Benchmarks }\end{array}$ & $\%$ Cases & $\begin{array}{c}\text { \% Improve- } \\
\text { ment }\end{array}$ & $\begin{array}{c}\text { No. of } \\
\text { Benchmarks }\end{array}$ & \% Cases \\
\hline II & 114 & 10 & 13.5 & 40 & 3 & 1.6 & 993 & 87 \\
\hline $\begin{array}{l}\text { Avg. Ejections } \\
\text { per instrn. }\end{array}$ & 338 & 29 & 858.7 & 79 & 7 & 858.0 & 730 & 63 \\
\hline
\end{tabular}

Table 4: Comparison of Enhanced Co-Scheduling with Slack Scheduling

(RecMII). Since Co-scheduling is basically Slack scheduling, fine-tuned for better selection of offset values, it is not surprising that the improvement, in terms of II, happens only in resource-critical loops. Also, it is possible that in some of the resource-critical loops both methods have achieved the MII. In a small number of benchmarks, Huff's Slack Scheduling achieves a better II, though the percentage improvement is only minor $(1.6 \%)$. This could be due to the order in which instructions are ejected in enhanced Co-scheduling.

The results shown in Table 4 exhibit a significant reduction in execution time (time to construct the schedules). In 988 of the benchmarks programs, the execution time of our Co-scheduling method is lower than that of Slack Scheduling. The average improvement in execution time is roughly by a factor of 4.5. As mentioned earlier, the execution time of our enhanced Co-scheduling method did not include the time to construct the MS-state diagram since these diagrams can be precomputed and stored in the compiler.

Apart from II and the time to construct schedule, we compare the two methods in terms of two other measures, namely (i) the average number of schedule slots tried (trials) per operation, and (ii) the average number of ejections per instruction. From Table 4, we observe that our enhanced Co-scheduling method performs better by a factor of 5 in $30 \%$ to $60 \%$ of the benchmarks. Lastly, even though Huff's Slack Scheduling achieves comparable improvement in performance, in terms of average ejections, trials, and scheduling time, the number of benchmarks where the improvement is observed is significantly lower (respectively 79, 5, and 158 benchmark programs).

\subsection{Comparison with Co-Scheduling}

Lastly, we compare the performance of the enhanced Co-scheduling method with the original method [8] in Table 5. Since the original Co-scheduling method considered only a single offset set, in more than $23 \%$ of benchmarks the enhanced method obtained lower II. The average improvement in II is $16.2 \%$. Both methods show improve- ment, in terms of the average number of trials, average number of ejected operations, and execution time, in more or less equal number of benchmarks.

\section{Related Work}

Several software pipelining methods have been proposed in the literature $[1,4,10,12,13,18]$. These methods are based on a global modulo reservation table. A comprehensive survey of these works is provided by Rau and Fisher in [17]. As explained in the Introduction, one major drawback of these methods is their inefficiency. These methods make several trials before successfully placing an operation in the modulo reservation table. They do not make effective use of the well-developed classical pipeline theory $[3,11,15]$.

The approach proposed in $[2,14,16]$ uses a finite state automaton (FSA)-based instruction scheduling technique. These methods use ideas from the classical pipeline theory, especially the notion of forbidden and permissible latency sequences. However these methods deal with general instruction scheduling, and do not handle software pipelining, where each scheduled instruction is initiated once every II cycles. Co-scheduling [8] is a state diagram-based software pipelining method. In the Co-scheduling method, a single permissible latency sequence chosen from the MSstate diagram and the corresponding offset values were used to guide the software pipelining method.

Lastly, Eichenberger and Davidson proposed a method, which still relies on the use of global resource table, but reduces the cost of structural-hazard checking by reducing the machine description [5]. Their approach uses forbidden latency information to obtain a minimal representation for reservation tables of different function units. Their method is applicable to both instruction scheduling and modulo scheduling.

\section{Conclusions}

In this paper we present an enhanced Co-scheduling method that makes use of the state diagram based approach 


\begin{tabular}{|l|c|c|c||c|c|c|c|c|}
\hline \multirow{2}{*}{ Measure } & \multicolumn{2}{|c|}{ Enhanced Co-Scheduling Better } & \multicolumn{2}{c|}{ Original Co-Scheduling Better } & \multicolumn{2}{c|}{ Both Same } \\
\cline { 2 - 9 } & $\begin{array}{c}\text { No. of } \\
\text { Benchmarks }\end{array}$ & \% Cases & $\begin{array}{c}\text { \% Improve- } \\
\text { ment }\end{array}$ & $\begin{array}{c}\text { No. of } \\
\text { Benchmarks }\end{array}$ & \% Cases & $\begin{array}{c}\text { \% Impro- } \\
\text { ment }\end{array}$ & $\begin{array}{c}\text { No. of } \\
\text { Benchmarks }\end{array}$ & \% Cases \\
\hline II & 255 & 23 & 16.2 & 4 & 0.03 & 1.4 & 871 & 77 \\
\hline $\begin{array}{c}\text { Avg. Ejections } \\
\text { per Instrn. }\end{array}$ & 88 & 8 & 526.5 & 149 & 13 & 525.2 & 893 & 79 \\
\hline $\begin{array}{c}\text { Avg. Trials } \\
\text { per Instrn. }\end{array}$ & 144 & 13 & 24.6 & 97 & 9 & 24.7 & 889 & 79 \\
\hline Exec. Time & 651 & 58 & 50.3 & 476 & 42 & 50.3 & 5 & 0 \\
\hline
\end{tabular}

Table 5: Comparison of Enhanced Co-scheduling with Original Co-scheduling

for software pipelining. This work identifies and eliminates redundant information in the original MS-state diagram. Based on the reduced MS-state diagram, we extend the original Co-scheduling method by considering multiple latency sequences. Experimental results reveal that the reduced states results in significant reduction ( 2 to 3 orders of magnitudes) in the number of paths. The enhanced Coscheduling was successful in constructing efficient schedules: they were better in terms of both II and the time to construct the schedule compared to the schedules constructed either by Huff's Slack scheduling method or by the original Co-scheduling method.

\section{Acknowledgments}

We wish to thank Sean Ryan, Chihong Zhang, members of CAPSL, University of Delaware, and the anonymous referees for their helpful suggestions. The last author acknowledges the support of National Science Foundation, U.S.A.

\section{References}

[1] E. R. Altman, R. Govindarajan, and G. R. Gao. Scheduling and mapping: Software pipelining in the presence of structural hazards. In Proc. of the ACM SIGPLAN '95 Conf. on Programming Language Design and Implementation, pages 139-150, La Jolla, CA, June 1995.

[2] V. Bala and N. Rubin. Efficient instruction scheduling using finite state automata. In Proc. of the 28th Ann. Intl. Symp. on Microarchitecture, pages 46-56, Ann Arbor, MI, Dec. 1995.

[3] E.S. Davidson, L.E. Shar, A.T. Thomas, and J.H. Patel. Effective control for pipelined computers. In Digest of Papers, 15th IEEE Computer Society Intl. Conf., COMPCON Spring '75. Feb. 1975.

[4] J. C. Dehnert and R. A. Towle. Compiling for Cydra 5. Journal of Supercomputing, 7:181-227, May 1993.

[5] A.E. Eichenberger and E.S. Davidson. A reduced multipipeline machine description that preserves scheduling constraints. In Proc. of the ACM SIGPLAN '96 Conf. on Programming Language Design and Implementation, Philadelphia, PA, May 1996.

[6] J. A. Fisher. Trace scheduling: A technique for global microcode compaction. IEEE Transactions on Computers, 7(30):478-490, July 1981.
[7] P. B. Gibbons and S. S. Muchnick. Efficient instruction scheduling for a pipelined architecture. In Proc. of the SIGPLAN '86 Symp. on Compiler Construction, pages 11-16, Palo Alto, CA, June 25-27, 1986.

[8] R. Govindarajan, E. R. Altman, and G. R. Gao. Co-scheduling hardware and software pipelines. In Proc. of the Second Intl. Symp. on High-Performance Computer Architecture, pages 52-61, San Jose, CA, Feb. 1996.

[9] R. Govindarajan, N.S.S. Narasimha Rao, E. R. Altman, and G. R. Gao. Software Pipelining using Reduced MS-State Diagrams. Technical Memo, Dept. of Computer Science \& Automation, Indian Institute of Science, Bangalore, India, Feb. 1997. (available via http://www.csa.iisc.ernet.in/ govind/papers/TR-97-2.ps.gz)

[10] R. A. Huff. Lifetime-sensitive modulo scheduling. In Proc. of the ACM SIGPLAN '93 Conf. on Programming Language Design and Implementation, pages 258-267, Albuquerque, NM, June 1993.

[11] P. M. Kogge. The Architecture of Pipelined Computers. McGrawHill Book Company, New York, New York, 1981.

[12] M. Lam. Software pipelining: An effective scheduling technique for VLIW machines. In Proc. of the SIGPLAN'88 Conf. on Programming Language Design and Implementation, pages 318-328, Atlanta, GA, June 1988.

[13] J. Llosa, M. Valero, E. Ayguadé, and A. González. Hypernode reduction modulo scheduling. In Proc. of the 28th Ann. Intl. Symp. on Microarchitecture, pages 350-360, Ann Arbor, MI, Dec. 1995.

[14] T. Muller. Employing finite state automata for resource scheduling. In Proc. of the 26th Ann. Intl. Symp. on Microarchitecture, Austin, TX, Dec. 1993.

[15] J. H. Patel and E. S. Davidson. Improving the throughput of a pipeline by insertion of delays. In Proc. of the 3rd Ann. Symp. on Computer Architecture, pages 159-164, Clearwater, FL, January 19-21, 1976.

[16] T. A. Proebsting and C. W. Fraser. Detecting pipeline structural hazards quickly. In Conf. Rec. of the 21st ACM SIGPLAN-SIGACT Symp. on Principles of Programming Languages, pages 280-286, Portland, OR, Jan. 1994.

[17] B. R. Rau and J. A. Fisher. Instruction-level parallel processing: History, overview and perspective. Journal of Supercomputing, 7:9-50, May 1993.

[18] B. R. Rau. Iterative modulo scheduling: An algorithm for software pipelining loops. In Proc. of the 27th Ann. Intl. Symp. on Microarchitecture, pages 63-74, San Jose, CA, Dec. 1994. 